\title{
Lung base CT findings in COVID-19 adult patients presenting with acute abdominal complaints: case series from a major New York City health system
}

\author{
Michael J. King ${ }^{1} \cdot$ Sara Lewis ${ }^{1,2}$ (D) Maria El Homsi ${ }^{1} \cdot$ Gabriela Hernandez Meza ${ }^{1}$ - Adam Bernheim ${ }^{1} \cdot$ Adam Jacobi $^{1}$. \\ Michael Chung ${ }^{1} \cdot$ Bachir Taouli ${ }^{1,2}$
}

Received: 2 May 2020 / Revised: 6 June 2020 / Accepted: 17 June 2020 / Published online: 4 July 2020

(C) European Society of Radiology 2020

\begin{abstract}
Objective To describe demographic, clinical, and lung base $\mathrm{CT}$ findings in COVID-19 patients presenting with abdominal complaints. Methods In this retrospective study, 76 COVID-19 patients who underwent abdominal CT for abdominal complaints from March 1 to April 15, 2020, in a large urban multihospital Health System were included. Those with positive abdominal CT findings $(n=14)$ were then excluded, with 62 patients undergoing final analysis $(30 \mathrm{M} / 32 \mathrm{~F}$; median age 63 years, interquartile range (IQR) $52-75$ years, range 30-90 years). Demographic and clinical data were extracted. CT lung base assessment was performed by a cardiothoracic radiologist. Data were compared between discharged and hospitalised patients using Wilcoxon or Fisher's exact tests.

Results The majority of the population was non-elderly $(56.4 \%,<65$ years) and most $(81 \%)$ had underlying health conditions. Nineteen percent were discharged and $81 \%$ were hospitalised. The most frequent abdominal symptoms were pain (83.9\%) and nausea/vomiting/anorexia (46.8\%). Lung base CT findings included ground-glass opacities (95.2\%) in a multifocal (95.2\%) and peripheral $(66.1 \%)$ distribution. Elevated laboratory values (when available) included C-reactive protein (CRP) (97.3\%), Ddimer (79.4\%), and ferritin ( $68.8 \%$ of males and $81.8 \%$ of females). Older age $(p=0.045)$, hypertension $(p=0.019)$, and lower haemoglobin in women $(p=0.042)$ were more frequent in hospitalised patients. There was no difference in lung base CT findings between discharged and hospitalised patients $(p>0.165)$.

Conclusions COVID-19 patients can present with abdominal symptoms, especially in non-elderly patients with underlying health conditions. Lung base findings on abdominal CT are consistent with published reports. Radiologists should be aware of atypical presentations of COVID-19.

Key Points

- COVID-19 infected patients can present with acute abdominal symptoms, especially in non-elderly patients with underlying health conditions, and may frequently require hospitalisation (81\%).

- There was no difference in lung base CT findings between patients who were discharged and those who were hospitalised.

- Lung base CT findings included multifocal and peripheral ground-glass opacities, consistent with published reports.
\end{abstract}

Sara Lewis

Sara.Lewis@mountsinai.org

Department of Diagnostic, Molecular and Interventional Radiology, Icahn School of Medicine at Mount Sinai, One Gustave L. Levy

Place, Box 1234, New York, NY 10029-6574, USA
2 BioMedical Engineering and Imaging Institute, Icahn School of Medicine at Mount Sinai, One Gustave L. Levy Place, Box 1234, New York, NY 10029-6574, USA 
Keywords Coronavirus $\cdot$ Multidetector computed tomography $\cdot$ Outcome assessment $\cdot$ Health care

$\begin{array}{ll}\text { Abbreviations } & \\ \text { CT } & \text { Computed tomography } \\ \text { COVID-19 } & \text { Coronavirus disease 2019 } \\ \text { ED } & \text { Emergency department } \\ \text { GGO } & \text { Ground-glass opacity } \\ \text { HIPAA } & \begin{array}{l}\text { Health Insurance Portability } \\ \text { and Accountability Act }\end{array} \\ \text { ICU } & \begin{array}{l}\text { Intensive care unit } \\ \text { IQR }\end{array} \\ \text { RT-PCR } & \begin{array}{l}\text { Reverquartile range } \\ \text { chain reaction }\end{array} \\ \text { SARS-CoV-2 } & \begin{array}{l}\text { Severe acute respiratory syndrome } \\ \text { coronavirus 2 }\end{array} \\ \text { WHO } & \text { World Health Organization }\end{array}$

\section{Introduction}

Following its initial discovery in December 2019 in Wuhan, China, the severe acute respiratory syndrome coronavirus 2 (SARS-CoV-2) was declared a global pandemic by the World Health Organization (WHO) on March 11, 2020 [1]. SARSCoV-2, transmitted between symptomatic and asymptomatic humans through respiratory droplets and direct contact, can result in severe pneumonia, acute respiratory distress syndrome (ARDS), and multiorgan failure [2]. Coronavirus disease 2019 (COVID-19), the term for the clinical illness caused by SARSCoV-2, has placed immense stress on the global healthcare apparatus and has resulted in profound economic disruption. As of 2 June 2020, there have been approximately 6 million confirmed cases and 371,000 deaths worldwide, with an estimated \$2.7 trillion in global economic losses [3, 4]. Approximately 1.9 million of those cases and 107,000 of those deaths are within the USA, with 372,000 cases and 24,000 deaths within New York State [3]. Currently, there is no specific anti-viral therapy or vaccine available for COVID-19.

Although typically characterised by fever and respiratory symptoms, gastrointestinal (GI) and/or genitourinary (GU) complaints as an initial presentation of COVID-19 have recently been described, albeit to a lesser extent [5-10]. In a metaanalysis of 69 studies including 4302 COVID-19 patients in Asia, $17.6 \%$ of patients reported GI symptoms [11]. Viral RNA was detected in stool samples from $48.1 \%$ of these patients [11]. A multicentre study from China with 204 patients showed that $50.5 \%$ reported GI symptoms on presentation [12]. Interestingly, and as we have observed clinically within our major multihospital Health System in New York City (NYC), many COVID-19 patients present with abdominal symptoms either in isolation or in combination with respiratory complaints. These patients commonly undergo computed tomography (CT), with incidental findings of peripheral ground-glass opacities (GGOs) and/or consolidative opacities noted at the lung bases, which have been described in the setting of COVID-19 [13-16].

Data is rapidly emerging given the novel nature of this devastating disease, with a paucity of published reports investigating abdominal presenting symptoms and subsequent CT imaging findings in COVID-19 patients. It remains to be determined how certain clinical and demographic factors including ethnic minority status, advanced age, or a lower income neighbourhood in NYC impact imaging findings or outcomes. To our knowledge, only a few small case series and case reports have been published on this topic to date [17-20].

The purpose of our study is to describe the demographic, clinical, biological, and lung base CT findings in COVID-19 patients presenting with acute abdominal complaints, either in isolation or in combination with respiratory complaints.

\section{Materials and methods}

\section{Patients}

This retrospective, Health Insurance Portability and Accountability Act (HIPAA)-compliant study was approved by our institutional review board with exemption for patient consent. The Department of Radiology imaging database was queried for abdominal or abdominopelvic CT examinations performed out of the emergency department (ED) throughout the hospitals in our Health System located within NYC (including 6 hospitals in Manhattan) between March 1, 2020, and April 15, 2020, using the search term 'viral pneumonia'. Inclusion criteria were the following: (1) patient age > 20 years; (2) acute abdominal symptoms (with or without respiratory complaints); (3) patients who underwent abdominal or abdominopelvic CT for evaluation of their symptoms; and (4) patients with confirmed positive COVID-19 reverse transcriptase polymerase chain reaction (RT-PCR) nasopharyngeal swab test obtained during the same ED visit. Exclusion criteria were the following: (1) lack of a positive COVID-19 RT-PCR test or (2) incomplete clinical information. The initial patient population consisted of 150 patients, with 74 cases excluded for various reasons (Fig. 1), resulting in a remaining population of 76 patients $(37 \mathrm{M} / 39 \mathrm{~F}$, median age 61.5 years, IQR 51-74 years, range 20-90 years).

Demographic and clinical data was recorded for each patient from the electronic medical records (EMR). Patients were stratified by age, sex, race, ethnicity, and NYC borough of residence. The following presenting symptoms were recorded: fever (oral temperature at ED presentation $\geq 37.0$ ${ }^{\circ} \mathrm{C}$ ), abdominal pain, nausea/vomiting/anorexia, diarrhoea, dysuria/haematuria/oliguria, cough, shortness of breath, chest 
Fig. 1 Flowchart of the study population

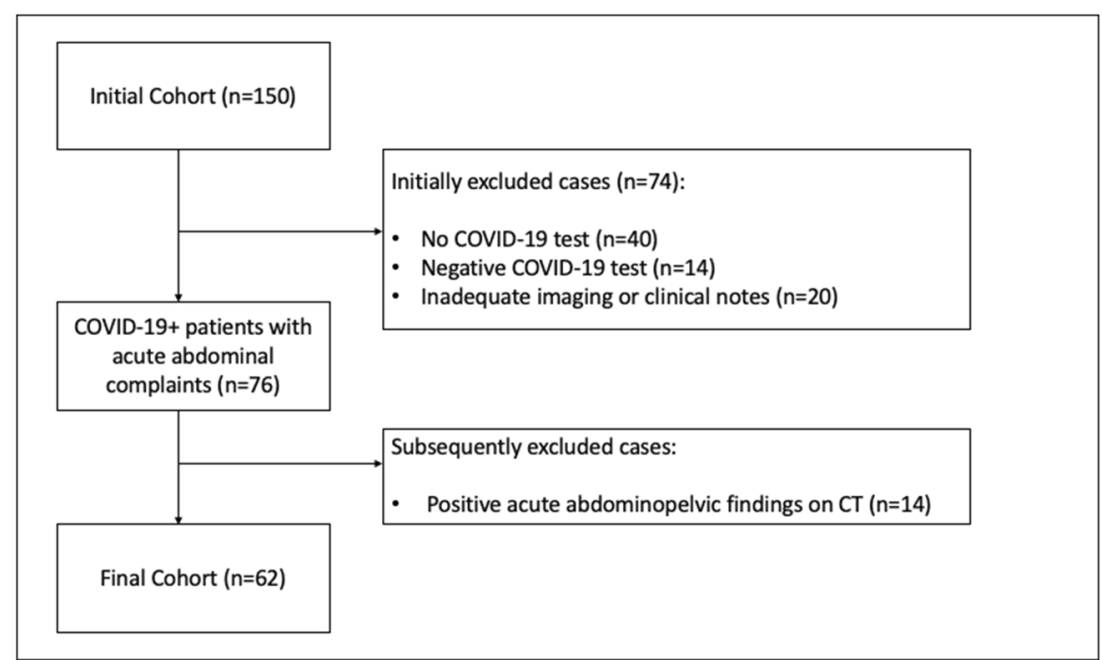

pain, symptoms commonly attributable to viral syndromes (including myalgia, malaise, fatigue, headache, rhinorrhoea, and sore throat), and other symptoms (including syncope, weakness, dizziness, and altered mental status). The following underlying medical conditions were recorded: obesity (defined as body mass index (BMI) $\geq 30 \mathrm{~kg} / \mathrm{m}^{2}$ ), hypertension, diabetes mellitus, cardiovascular disease (including coronary artery disease, cerebrovascular disease, peripheral vascular disease, and/or congestive heart failure), chronic renal disease, asthma/chronic obstructive pulmonary disease (COPD), and any cancer history or other reasons for immunosuppression. Blood laboratory values were recorded, including aspartate aminotransferase (AST), alanine aminotransferase (ALT), Creactive protein (CRP), procalcitonin, D-dimer, ferritin, lipase, white blood cell (WBC) count, lymphocyte percentage, and haemoglobin $(\mathrm{Hb})$.

\section{Image acquisition}

Abdominal CT examinations were performed at several EDs across our Health System using clinically available platforms, including GE Medical Systems Revolution ( $n=12)$, GE Medical Systems Lightspeed VCT $(n=20)$, Siemens Somatom $(n=16)$, and Toshiba Aquilion $(n=14)$ scanners. The CT examinations were performed on the abdomen or the abdomen and pelvis, with $(n=39)$ or without $(n=23)$ Isovue370 intravenous contrast (iopamidol 76\%, Bracco Diagnostics), in the supine or prone position depending on initial patient complaint. CT scanning parameters were as follows: matrix size of $512 \times 512, \mathrm{kVp}$ values ranging from 80 to $140 \mathrm{mSv}$, slice thickness reconstructed at $3 \mathrm{~mm}$, and pixel spacing (in-plane resolution) range of $0.596-0.977 \mathrm{~mm}$ in the axial plane. Coronal and sagittal reconstructions were generated automatically by the scanners. Lung base images were reconstructed using a lung kernel with reconstructed slice thickness of $3 \mathrm{~mm}$.

\section{Image analysis}

The abdominal CT examinations were reviewed by two board-certified fellowship-trained abdominal radiologists (M.K. and S.L.) in consensus with 2 and 10 years of experience, respectively, using PACS (Centricity 3.0, General Electric Medical Systems), to confirm the absence/presence of findings in the abdomen that could explain the clinical symptoms. The lung base findings were then independently reviewed by a board-certified fellowship-trained cardiothoracic radiologist (M.C.) with 5 years of experience, who was aware of the presence of COVID-19 diagnosis but blinded to clinical symptoms. The lung bases were qualitatively assessed for the presence or absence of the following findings: groundglass opacities (GGOs), consolidation, lesions with a rounded morphology, septal thickening, 'crazy paving' pattern, peripheral distribution, lung base multifocal involvement, bilateral involvement, and pleural effusion. Two patients underwent chest CT at the same time as their abdominal CT. In both cases, the chest portion of the study was not evaluated, and only the lung base findings on the abdominal scan were included in our analysis for consistency.

\section{Study endpoints}

The following patient outcomes were recorded: (1) discharge from the ED (mild disease); (2) hospitalisation, including admission to an intensive care unit (ICU); and (3) death. For patients who were admitted, the duration of hospitalisation or the time from admission to death was recorded. A final review of the patient's EMR was undertaken on April 24, 2020. 


\section{Statistical analysis}

Demographic, clinical, biological, and imaging data were summarised using descriptive statistics. The Wilcoxon test was used for continuous variables. Fisher's exact test was used for categorical variables. Data were compared between groups of patients who were either discharged from the ED or hospitalised (including patients who died). A $p$ value $\leq 0.05$ represented an acceptable level of statistical significance in our study. All statistical tests were conducted using R statistical software (version 3.6.3).

\section{Results}

\section{Patients}

Of the initially included patients $(n=76)$, those found to have a positive CT finding within the abdomen or pelvis $(n=14)$ were excluded from analysis. The final study population comprised 62 patients $(30 \mathrm{M} / 32 \mathrm{~F}$; median age 63 years, interquartile range (IQR) $52-75$ years, range $30-90$ years). The fourteen patients who were excluded due to the presence of positive findings within the abdomen or pelvis at CT had GI diagnoses (oesophagitis $(n=1)$, perforated duodenal ulcer $(n=$ $1)$, small bowel enteritis $(n=1)$, small bowel obstruction $(n=$ $1)$, large bowel obstruction $(n=1)$, colitis $(n=2)$, perianal abscess $(n=1)$, biliary distention $(n=1)$, cholelithiasis $(n=1)$, or acute pancreatitis $(n=1))$ or GU diagnoses (nephrolithiasis and/or hydronephrosis $(n=5)$, pyelonephritis $(n=1)$, or postcystectomy fluid collection $(n=1))$. Four patients had both GI and $\mathrm{GU}$ diagnoses.

\section{Demographic and clinical findings}

Demographic and clinical characteristics of the study population are summarised in Table 1 . Most patients lived in Manhattan $(45 \%, n=28 / 62)$ and a majority $(81 \%, n=50 /$ 62 ) had at least one underlying medical condition. The median time interval between scanning and confirmed COVID-19 diagnosis via RT-PCR was 0.5 days (IQR $0-1$ ).

Patients were stratified by demographic, clinical, biological, and lung base $\mathrm{CT}$ findings according to patient outcomes. In our study sample, $19 \%(n=12 / 62)$ of patients were discharged from the ED without hospital admission, while $81 \%(n=50 / 62)$ were hospitalised. Of the hospitalised patients, $10 \%(n=6 / 62)$ died while the remaining $71 \%(n=$ $44 / 62$ ) were discharged. The 6 patients who died had a median age of 69 years (range 58-83 years); 1 died of cardiopulmonary arrest with COVID-19 pneumonia and diabetes listed as contributing factors despite attempted resuscitation, 4 died of COVID-19 respiratory failure in the setting of a do not resuscitate/do not intubate (DNR/DNI) order, and 1 died of
COVID-19 respiratory failure and acute renal failure in the setting of a DNR/DNI order. No patients were ventilated or admitted to the ICU. Of those hospitalised, the median duration of hospitalisation (until discharge or death) was 5.0 days (IQR 3.0-9.0 days). The hospitalised patients were older than those discharged from the ED (median age 63.0 years (IQR $55.8-77.8$ years) vs. 54.5 years (IQR 46.0-65.0 years), $p=$ 0.045 , respectively). The hospitalised patients were managed clinically at the discretion of the medical teams, and none received subsequent abdominal imaging throughout their hospital stay. There was no difference in sex or BMI (all $p$ values $>0.844$ ) between the 2 groups. Hypertension was more frequent in hospitalised patients $(p=0.019)$, whereas cough $(p=$ $0.044)$ and shortness of breath $(p=0.038)$ were more frequent in those discharged from the ED. Otherwise, there was no significant difference in the frequency of presenting symptoms (all $p$ values $>0.116$ ) or underlying health conditions (all $p$ values $>0.085$ ) (Table 1).

\section{Blood laboratory results}

The median and interquartile ranges of selected blood laboratory values (when available) are summarised in Table 2 . Although a majority $(57.4 \%, n=31 / 54)$ of patients demonstrated a mildly elevated AST, the most frequent abnormalities were acute markers of inflammation. Median haemoglobin was lower for female patients hospitalised compared with those discharged from the ED (median $\mathrm{Hb} 11.8 \mathrm{~g} / \mathrm{DL}$ (IQR $10.9-13.4$ ) vs. $13.6 \mathrm{~g} / \mathrm{DL}$ (IQR 12.9-14.1), $p=0.042$ ). There was no difference in other blood laboratory values between the 2 patient groups (all $p$ values $>0.087$ ). With regard to elevated D-dimer, no patients demonstrated acute thrombotic events at the time of imaging.

\section{Imaging results}

A summary of the lung base CT findings in the whole patient population and stratified by outcome is described in Table 3 . All patients demonstrated at least one lung base finding on abdominal CT. An overwhelming majority of patients demonstrated GGOs (95.2\%), with findings described in a multifocal $(95.2 \%)$ and bilateral (93.6\%) pattern (Figs. 2 and 3). Only 1 patient $(1.6 \%)$ demonstrated pleural effusion. There was no significant difference in the frequency of imaging findings at the lung bases between the discharged and hospitalised groups $(p>0.165)$.

\section{Discussion}

In this study, we described demographic, clinical, biological, and lung base CT findings in a population of COVID-19 patients who presented to our Health System with acute 
Table 1 Demographic and clinical characteristics of patients with COVID-19 in relation to outcomes (discharged vs hospitalised). Values are given as numerical value with percentages (percent relative to total number in the respective patient group) unless otherwise stated

\begin{tabular}{|c|c|c|c|c|}
\hline Characteristic & All patients $(n=62)$ & Discharged from ED $(n=12)$ & Hospitalised $(n=50)$ & $p$ \\
\hline Age (year) & $62.5(52.3-75.0)^{\#}$ & $54.5(46.0-65.0)^{\#}$ & $63.0(55.8-77.8)^{\#}$ & 0.045 \\
\hline $21-49$ years & $12(19.4 \%)$ & $4(33 \%)$ & $8(16 \%)$ & \\
\hline $50-64$ years & $23(37.1 \%)$ & $5(42 \%)$ & $18(36 \%)$ & \\
\hline$\geq 65$ years & $27(43.5 \%)$ & $3(25 \%)$ & $24(48 \%)$ & \\
\hline \multicolumn{4}{|l|}{ Sex } & 0.844 \\
\hline Female & $32(51.6 \%)$ & $7(58 \%)$ & $25(50 \%)$ & \\
\hline Male & $30(48.4 \%)$ & $5(42 \%)$ & $25(50 \%)$ & \\
\hline Body mass index (BMI) $\left(\mathrm{kg} / \mathrm{m}^{2}\right)$ & $27(24-31)^{\#}$ & $28(25-28.5)^{\#}$ & $27(24-31)^{\#}$ & 0.879 \\
\hline \multicolumn{4}{|l|}{ Race } & 0.697 \\
\hline White & $29(46.8 \%)$ & $5(42 \%)$ & $24(48 \%)$ & \\
\hline African American & $19(30.7 \%)$ & $4(33 \%)$ & $15(30 \%)$ & \\
\hline Asian & $2(3.2 \%)$ & $1(8 \%)$ & $1(2 \%)$ & \\
\hline Other/unknown & $12(19.3 \%)$ & $2(17 \%)$ & $10(20 \%)$ & \\
\hline \multicolumn{4}{|l|}{ Ethnicity } & 0.912 \\
\hline Hispanic & $20(32.3 \%)$ & $3(25 \%)$ & $17(34 \%)$ & \\
\hline Non-Hispanic & $30(48.4 \%)$ & $7(58 \%)$ & $23(46 \%)$ & \\
\hline Other/unknown & $12(19.3 \%)$ & $2(17 \%)$ & $10(20 \%)$ & \\
\hline \multicolumn{4}{|l|}{ Borough of residence } & 0.872 \\
\hline Manhattan & $28(45.2 \%)$ & $6(50 \%)$ & $22(44 \%)$ & \\
\hline Brooklyn & $11(17.7 \%)$ & $1(8 \%)$ & $10(20 \%)$ & \\
\hline Queens & $16(25.8 \%)$ & $4(33 \%)$ & $12(24 \%)$ & \\
\hline Bronx & $6(9.7 \%)$ & $1(8 \%)$ & $5(10 \%)$ & \\
\hline Staten Island & $0(0.0 \%)$ & $0(0 \%)$ & $0(0 \%)$ & \\
\hline Outside NYC & $1(1.6 \%)$ & $0(0 \%)$ & $1(2 \%)$ & \\
\hline \multicolumn{5}{|l|}{ Presenting symptom } \\
\hline Fever $\$$ & $29(46.8 \%)$ & $6(50 \%)$ & $23(46 \%)$ & 1 \\
\hline Abdominal pain & $52(83.9 \%)$ & $11(92 \%)$ & $41(82 \%)$ & 0.670 \\
\hline Nausea/vomiting/anorexia & $29(46.8 \%)$ & $3(24 \%)$ & $26(52 \%)$ & 0.116 \\
\hline Diarrheal & $16(25.8 \%)$ & $3(24 \%)$ & $13(26 \%)$ & 1 \\
\hline Dysuria/haematuria/oliguria & $7(11.3 \%)$ & $3(24 \%)$ & $4(8 \%)$ & 0.125 \\
\hline Cough & $12(19.4 \%)$ & $5(42 \%)$ & $7(14 \%)$ & 0.044 \\
\hline Shortness of breath & $8(12.9 \%)$ & $4(33 \%)$ & $4(8 \%)$ & 0.038 \\
\hline Chest pain & $4(6.5 \%)$ & $1(8 \%)$ & $3(6 \%)$ & 1 \\
\hline General viral syndromic symptoms* & $14(22.6 \%)$ & $4(33 \%)$ & $10(20 \%)$ & 0.266 \\
\hline Other $^{\wedge}$ & $8(12.9 \%)$ & $2(17 \%)$ & $6(12 \%)$ & 0.64 \\
\hline Underlying health conditions & $50(81 \%)$ & & & \\
\hline Obesity $\%$ & $19(30.7 \%)$ & $1(8 \%)$ & $18(36 \%)$ & 0.085 \\
\hline Hypertension & $36(58.1 \%)$ & $3(24 \%)$ & $33(66 \%)$ & 0.019 \\
\hline Diabetes mellitus & $19(30.7 \%)$ & $2(17 \%)$ & $17(34 \%)$ & 0.313 \\
\hline Cardiovascular disease & $11(17.7 \%)$ & $1(8 \%)$ & $10(20 \%)$ & 0.674 \\
\hline Chronic renal disease & $11(17.7 \%)$ & $0(0 \%)$ & $11(22 \%)$ & 0.102 \\
\hline Asthma/COPD & $13(21.0 \%)$ & $2(17 \%)$ & $11(22 \%)$ & 1 \\
\hline Cancer history & $10(16.1 \%)$ & $0(0 \%)$ & $10(20 \%)$ & 0.186 \\
\hline Immunosuppression & $3(4.8 \%)$ & $0(0 \%)$ & $3(6 \%)$ & 1 \\
\hline
\end{tabular}

Continuous variables (age and BMI) were assessed using Wilcoxon, while categorical variables (sex, race, ethnicity, borough of residence, presenting symptoms, and underlying health conditions) were tested using Fisher's exact test. Significant $p$ values are italicised

${ }^{\#}$ Value given as median (interquartile range)

${ }^{\$}$ Defined as oral temperature on presentation to the $\mathrm{ED} \geq 37.0^{\circ} \mathrm{C}$

*Includes myalgia, malaise, fatigue, headache, rhinorrhoea, sore throat

${ }^{\wedge}$ Includes syncope, weakness, dizziness, altered mental status

${ }^{\%}$ Defined as BMI $\geq 30 \mathrm{~kg} / \mathrm{m}^{2}$

abdominal complaints, either in isolation or in combination with respiratory complaints. Nineteen percent of the patients were discharged from the ED without hospital admission and $81 \%$ were hospitalised. Of those hospitalised, the median duration of hospitalisation (until discharge or death) was 5 days. Less than $10 \%$ of our patient population died, all of whom were admitted to the hospital, but not to the ICU. The higher median age observed in patients that died is consistent 
Table 2 Blood laboratory values in patients with COVID-19 in relation to outcomes (discharged vs hospitalised). Data between each patient group were compared using the Wilcoxon test. Values are given as median with interquartile range in parentheses unless otherwise stated. Reference ranges are given below each laboratory value name in brackets

\begin{tabular}{|c|c|c|c|c|c|}
\hline Laboratory value & All patients $(n=62)$ & $\begin{array}{l}\text { Patients with abnormal } \\
\text { lab values }(\%)\end{array}$ & $\begin{array}{l}\text { Discharged from ED } \\
(n=12)\end{array}$ & Hospitalised $(n=50)$ & $p$ \\
\hline Aspartate aminotransferase (AST) $[1-35 \mathrm{U} / \mathrm{L}]$ & $39.0(25.0-62.3)$ & $31 / 54(57.4 \%)$ & $37.0(25.5-53.3)$ & $41.5(25.0-70.0)$ & 0.385 \\
\hline Alanine transaminase (ALT) $[1-45 \mathrm{U} / \mathrm{L}]$ & $25.0(19.0-44.0)$ & $13 / 57(22.8 \%)$ & $25.0(18.5-45.5)$ & $25.5(19.0-42.3)$ & 0.911 \\
\hline C-reactive protein (CRP) $[0-5.0 \mathrm{mg} / \mathrm{L}]$ & $67.8(35.9-99.3)$ & $36 / 37(97.3 \%)$ & $56.4(28.9-77.6)$ & $68.8(36.3-107.0)$ & 0.522 \\
\hline Procalcitonin $[0-0.49 \mathrm{ng} / \mathrm{mL}]$ & $0.1(0.1-0.4)$ & $8 / 39(20.5 \%)$ & $0.3(0.2-0.4)$ & $0.1(0.1-0.3)$ & 0.632 \\
\hline D-dimer $[0-0.5 \mu \mathrm{g} / \mathrm{mL}]$ & $1.0(0.7-1.7)$ & $27 / 31(79.4 \%)$ & $7.0(0.4-1.0)$ & $1.1(0.7-2.3)$ & 0.323 \\
\hline Ferritin (males) ${ }^{\#}[30-400 \mathrm{ng} / \mathrm{mL}]$ & $740.5(387.8-1994.3)$ & $11 / 16(68.8 \%)$ & NA & $740.5(387.8-1994.3)$ & NA \\
\hline Ferritin (females) $[15-150 \mathrm{ng} / \mathrm{mL}]$ & $391.5(248.0-626.3)$ & $18 / 22(81.8 \%)$ & $346.0(322.5-269.5)$ & $422.5(225.3-656.3)$ & 0.689 \\
\hline Lipase [8-78 U/L] & $29.5(16.8-71.3)$ & $12 / 52(23.1 \%)$ & $31.0(22.3-44.8)$ & $29.5(16.3-75.8)$ & 0.972 \\
\hline White blood cell count (WBC) $[4.5-11.0 \mathrm{~K} / \mu \mathrm{L}]$ & $6.7(5.6-8.9)$ & $15 / 62(24.2 \%)^{*}$ & $6.0(5.0-6.7)$ & $7.1(5.6-9.4)$ & 0.090 \\
\hline Lymphocyte \% [15-50] & $16.6(11.1-23.3)$ & $27 / 62(43.5 \%)^{\wedge}$ & $21.6(15.9-24.3)$ & $15.2(10.6-22.9)$ & 0.087 \\
\hline Haemoglobin $(\mathrm{Hb})$ (males) [13.9-16.3 g/D] & $14.3(12.7-15.7)$ & $12 / 29(41.4 \%)^{\&}$ & $15.4(15.0-15.7)$ & $14.2(12.4-15.6)$ & 0.201 \\
\hline Haemoglobin (Hb) (females) [11.7-15.0 g/DL] & $12.4(11.1-13.7)$ & $11 / 32(34.3 \%)^{\&}$ & $13.6(12.9-14.1)$ & $11.8(10.9-13.4)$ & 0.042 \\
\hline
\end{tabular}

No patients were admitted to the ICU. Not all laboratory tests were available in all patients. $N A$ not applicable. Significant $p$ values are italicised

*Includes patients with $\mathrm{WBC}<4.5 \mathrm{~K} / \mu \mathrm{L}$ and $>11.0 \mathrm{~K} / \mu \mathrm{L}$

^Includes only patients with lymphocyte $\%<15$

${ }^{\&}$ Includes only patients with $\mathrm{Hb}<13.9 \mathrm{~g} / \mathrm{D}$ (males) and $11.7 \mathrm{~g} / \mathrm{D}$ (females)

${ }^{\text {\# }}$ Results available only for hospitalised patients

with anecdotal evidence and with one of the earliest and largest published clinical reports on COVID-19 from Wuhan, China [5]. Interestingly, our 0\% ICU admission rate is in line with very recent reports - including one from a neighbouring academic institution in NYC consisting of 278 patientssupporting an emerging concept that patients presenting with GI complaints may have milder disease severity with lower ICU admission and mortality rates [7, 8]. However, these findings contrast with a study of 204 Chinese patients with GI symptoms that demonstrated a $7.8 \%$ ICU admission rate [12]. Our mortality rate is in line with other reported mortality rates for COVID-19, which vary between 1.4 and 28\% depending on the series $[5,9,21,22]$.
Most patients $(81 \%, n=50 / 62)$ in our study had underlying medical conditions, which likely mirrors the US public health situation. The only risk factor associated with need for hospitalisation was hypertension, contrasting with a recent Chinese report that suggested diabetes be considered a poor prognostic risk factor in COVID-19 [23]. A recent report of 107 patients suggested that lymphopenia is an indicator of disease severity in COVID-19 patients [24]. The most frequent laboratory abnormalities in our study were acute markers of inflammation (including CRP, D-dimer, and ferritin); however, the only significant difference in laboratory values was for hospitalised women having a lower haemoglobin compared with those discharged from the ED.
Table 3 Lung base findings on abdominal $\mathrm{CT}$ in 62 patients with COVID-19 in relation to outcomes (discharged vs hospitalised). Data between each patient group were compared using Fisher's exact test. Values are given as numerical value with percentages (percent relative to total number in the respective patient group)

\begin{tabular}{lllll}
\hline Finding & All patients $(n=62)$ & $\begin{array}{l}\text { Discharged from ED } \\
(n=12)\end{array}$ & Hospitalised $(n=50)$ & $p$ \\
\hline Ground-glass opacities & $59(95.2 \%)$ & $12(60 \%)$ & $47(94 \%)$ & 1 \\
Multifocal involvement & $59(95.2 \%)$ & $11(92 \%)$ & $48(96 \%)$ & 0.482 \\
Bilateral involvement & $58(93.6 \%)$ & $10(83 \%)$ & $48(96 \%)$ & 0.165 \\
Peripheral distribution & $41(66.1 \%)$ & $10(83 \%)$ & $31(62 \%)$ & 0.195 \\
Consolidation & $29(46.8 \%)$ & $4(33 \%)$ & $25(50 \%)$ & 0.350 \\
Rounded morphology & $28(45.2 \%)$ & $6(50 \%)$ & $22(44 \%)$ & 0.755 \\
'Crazy paving' attenuation & $4(6.5 \%)$ & $0(0 \%)$ & $4(8 \%)$ & 0.578 \\
Septal thickening & $1(1.6 \%)$ & $0(0 \%)$ & $1(2 \%)$ & 1 \\
Pleural effusion & $1(1.6 \%)$ & $0(0 \%)$ & $1(2 \%)$ & 1 \\
\hline
\end{tabular}


Fig. 2 A 58-year-old male presenting with abdominal pain for 7 days, who was subsequently discovered to be COVID-19positive. (a) Axial and (b) coronal $\mathrm{CT}$ at the level of the lung bases show multifocal bilateral groundglass opacities with a rounded morphology (arrows); many of which are peripheral in distribution. This patient was hospitalised and ultimately died 10 days later from respiratory failure related to COVID-19

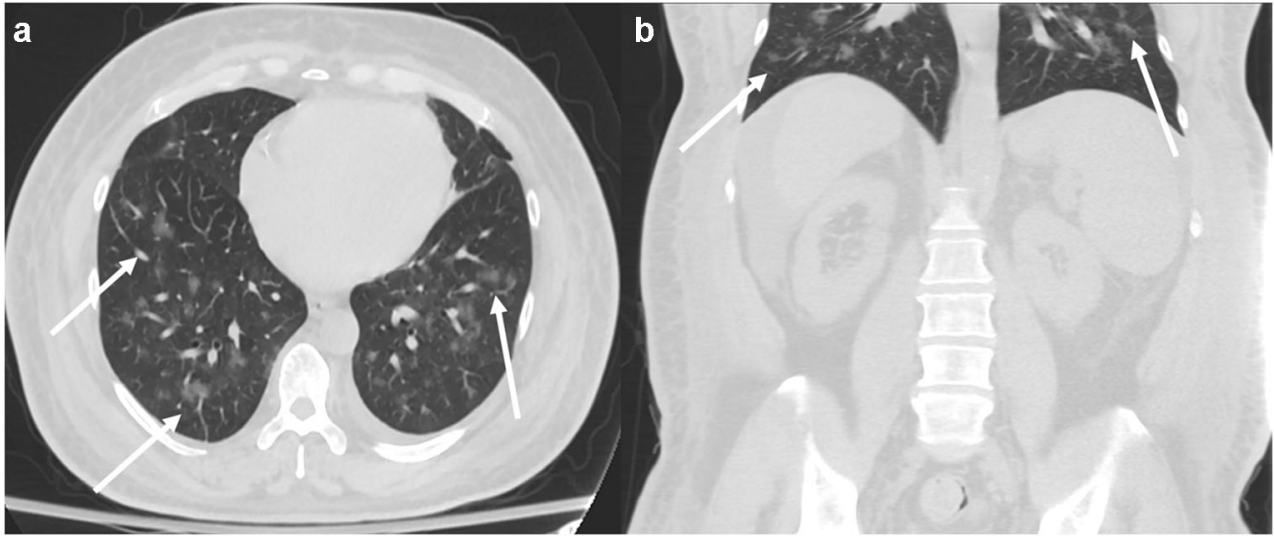

These discrepancies in our results compared with prior studies are likely due to our small sample size and differences in the study population.

Our lung base CT findings are consistent with numerous published reports on nonspecific imaging features that are typical for COVID-19, including ground-glass opacities and consolidation, often with a rounded morphology with a bilateral, peripheral, and/or multifocal distribution, while lymphadenopathy and pleural effusions are characteristically absent $[13,25-28]$. As our study suggests, the lower lobe predominance of these findings requires abdominal radiologists to keep COVID-19 diagnosis in mind even when patients present solely with abdominal complaints, and to suggest it as a differential diagnosis where appropriate. Interestingly, no differences were found between the frequencies of lung base CT findings between the two patient groups.

Understanding the exact mechanism of SARS-CoV-2 resulting in abdominal symptoms remains both interesting and challenging. Potential explanations include direct viral infection of the GI or GU systems, co-infection with another pathogen, or referred pain from infection at the lung bases [29]. Similar to the 2003 coronavirus SARS-CoV but with a much higher affinity, SARS-CoV-2 infection in humans depends on the ability of the virus to bind with the angiotensinconverting enzyme 2 (ACE2) receptor [30, 31]. ACE2 is expressed in the human lung, upper oesophagus, ileum, colon, renal proximal tubules, and urinary bladder, providing a conceivable explanation for viral involvement at these sites [32, 33]. Viral infection of enterocytes is likely to cause an alteration of intestinal permeability, leading to a spectrum of GI symptoms including abdominal pain, nausea/vomiting, and diarrhoea [34]. According to Roman et al, there is no clear description of autonomous nervous system involvement with COVID-19 at this point [35].

Given this, a study of 204 patients with acute respiratory illnesses with confirmed nasopharyngeal swabbing for viral pathogens, including human coronavirus, found that GI symptoms could not be explained by the presence of viral respiratory pathogens in stool samples [29]. Abdominal symptoms have been described in other closely related coronavirus infections,

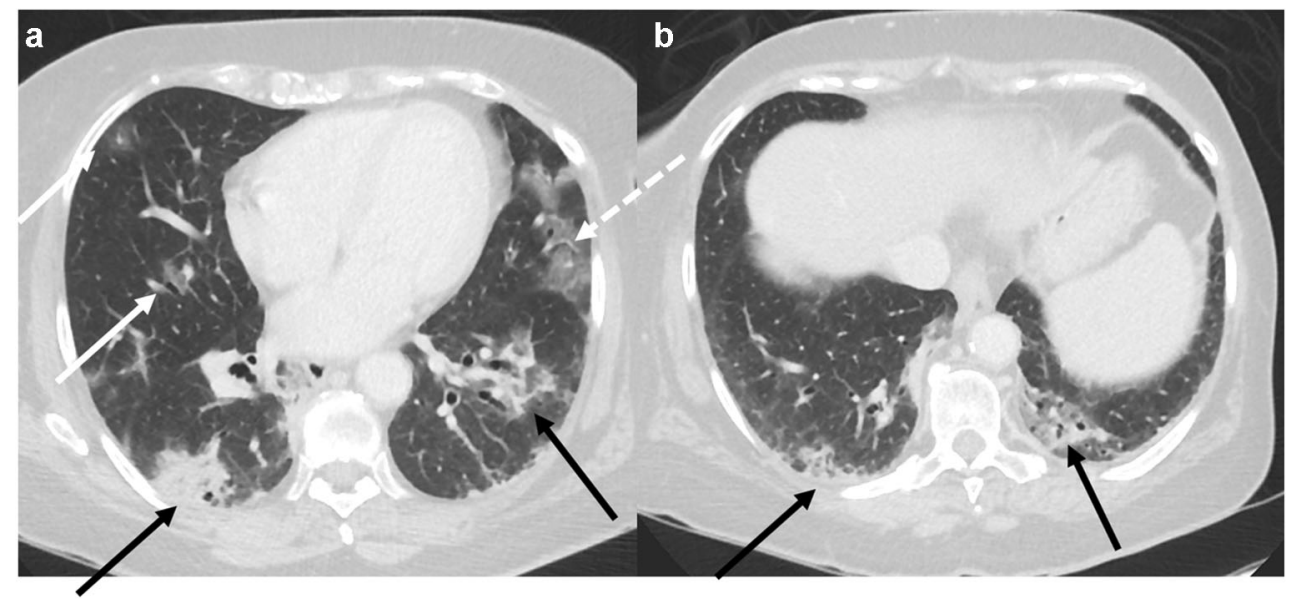

Fig. 3 A 63-year-old female with abdominal pain, nausea, and diarrhoea for 7 days, who was subsequently discovered to be COVID-19-positive. Axial CT of the lung bases at two different levels (a, b) shows predominantly peripheral, multifocal, and bilateral ground-glass (white arrows) and consolidative (black arrows) opacities with a region of 'crazy paving' attenuation (dashed arrow), a less commonly seen imaging finding in COVID-19. This patient was discharged home after being hospitalised for 8 days 
with GI symptoms and diarrhoea reported with high frequency (23-70\%) in severe acute respiratory syndrome (SARS) and in $>33 \%$ of patients with Middle Eastern respiratory syndrome (MERS) [36-38]. A recent preliminary, non-peer-reviewed study also suggested that ACE2 is also expressed in cholangiocytes, perhaps providing a mechanism for liver dysfunction and elevated serum liver enzymes [39]. Of note, there was mild elevation in serum AST in our study. The renal and urinary bladder expression of ACE2 may explain the urinary symptoms in our study sample. Further work is warranted to elucidate the mechanism of action in this regard.

Our study has several limitations. First, our data was obtained retrospectively from available data within the EMR, which may limit the generalisability of our results. Much of the clinical and radiologic literature published to date has been from Asia; more robust studies from the USA, a region with marked demographic and clinicopathologic differences (including higher rates of obesity and diabetes, for example), need to be performed in order to see how our results compare. We had incomplete race/ethnicity information, which has limited our ability to assess any meaningful associations related to these parameters. Additionally, future studies from the USA should incorporate longer follow-up periods and larger patient populations, including assessment for viral particles in stool and urine, an important clinical component missing in our analysis.

In conclusion, we report demographic, clinical, biological, and lung base CT findings in a group of patients presenting to our Health System with acute abdominal complaints who were found to have COVID-19. It is essential that the abdominal radiologist be aware that patients presenting with a wide variety of abdominal complaints may have COVID-19 infection in the absence of other findings in the abdomen or pelvis. Further work in a larger patient population is needed to evaluate these findings in relation to disease severity and outcome, especially given the urgency and widespread nature of COVID-19 infection.

Funding information The authors state that this work has not received any funding.

\section{Compliance with ethical standards}

Guarantor The scientific guarantor of this publication is Bachir Taouli, MD.

Conflict of interest The authors of this manuscript declare no relationships with any companies, whose products or services may be related to the subject matter of the article.

Statistics and biometry One of the authors has significant statistical expertise.

Informed consent Written informed consent was waived by the Institutional Review Board.
Ethical approval Institutional Review Board approval was obtained.

\section{Methodology}

- Retrospective

- Observational

- Performed at one health system comprised of 6 hospitals

\section{References}

1. Ducharme J (2020) World Health Organization Declares COVID19 a 'Pandemic.' Here's what that means. Time

2. Cheng Z, Lu Y, Cao Q et al (2020) Clinical features and chest CT manifestations of coronavirus disease 2019 (COVID-19) in a single-center study in Shanghai, China. AJR Am J Roentgenol. https://doi.org/10.2214/AJR.20.22959:1-6

3. Johns Hopkins University School of Engineering (2020) Coronavirus COVID-19 global cases by the center for systems science and engineering. https://coronavirus.jhu.edu/map.html. Last accessed 2 June 2020

4. Orlik T, Rush J, Cousin M, Hong J (2020) Coronavirus could cost the global economy $\$ 2.7$ trillion. Here's how. Bloomberg

5. Guan WJ, Ni ZY, Hu Y et al (2020) Clinical characteristics of coronavirus disease 2019 in China. N Engl J Med. https://doi.org/ 10.1056/NEJMoa2002032

6. Huang C, Wang Y, Li X et al (2020) Clinical features of patients infected with 2019 novel coronavirus in Wuhan, China. Lancet 395:497-506

7. Han C, Duan C, Zhang S et al (2020) Digestive symptoms in COVID-19 patients with mild disease severity: clinical presentation, stool viral RNA testing, and outcomes. Am J Gastroenterol. https://doi.org/10.14309/ajg.0000000000000664

8. Chen A, Agarwal A, Ravindran N, To C, Zhang T, Thuluvath PJ (2020) Gastrointestinal symptoms and COVID-19: case-control study from the United States. Gastroenterology. https://doi.org/10. 1053/j.gastro.2020.04.017

9. Richardson S, Hirsch JS, Narasimhan M et al (2020) Presenting characteristics, comorbidities, and outcomes among 5700 patients hospitalised with COVID-19 in the New York City area. JAMA. https://doi.org/10.1001/jama.2020.6775

10. Tian Y, Rong L, Nian W, He Y (2020) Review article: gastrointestinal features in COVID-19 and the possibility of faecal transmission. Aliment Pharmacol Ther 51:843-851

11. Cheung KS, Hung IF, Chan PP et al (2020) Gastrointestinal manifestations of SARS-CoV-2 infection and virus load in fecal samples from the Hong Kong cohort and systematic review and metaanalysis. Gastroenterology. https://doi.org/10.1053/j.gastro.2020. 03.065

12. Pan L, Mu M, Yang P et al (2020) Clinical characteristics of COVID-19 patients with digestive symptoms in Hubei, China: a descriptive, cross-sectional, multicenter study. Am J Gastroenterol. https://doi.org/10.14309/ajg.0000000000000620

13. Chung M, Bernheim A, Mei X et al (2020) CT imaging features of 2019 novel coronavirus (2019-nCoV). Radiology 295:202-207

14. Caruso D, Zerunian M, Polici M et al (2020) Chest CT features of COVID-19 in Rome, Italy. Radiology. https://doi.org/10.1148/ radiol.2020201237:201237

15. Xu YH, Dong JH, An WM et al (2020) Clinical and computed tomographic imaging features of novel coronavirus pneumonia caused by SARS-CoV-2. J Infect 80:394 400

16. Shi H, Han X, Jiang N et al (2020) Radiological findings from 81 patients with COVID-19 pneumonia in Wuhan, China: a descriptive study. Lancet Infect Dis 20:425-434 
17. Siegel A, Chang PJ, Jarou ZJ et al (2020) Lung base findings of coronavirus disease (COVID-19) on abdominal CT in patients with predominant gastrointestinal symptoms. AJR Am J Roentgenol. https://doi.org/10.2214/AJR.20.23232:1-3

18. Kim J, Thomsen T, Sell N, Goldsmith AJ (2020) Abdominal and testicular pain: an atypical presentation of COVID-19. Am J Emerg Med. https://doi.org/10.1016/j.ajem.2020.03.052

19. Tay HS, Harwood R (2020) Atypical presentation of COVID-19 in a frail older person. Age Ageing. https://doi.org/10.1093/ageing/ afaa068

20. Dane B, Brusca-Augello G, Kim D, Katz DS (2020) Unexpected findings of coronavirus disease (COVID-19) at the lung bases on abdominopelvic CT. AJR Am J Roentgenol. https://doi.org/10. 2214/AJR.20.23240:1-4

21. Wu Z, McGoogan JM (2020) Characteristics of and important lessons from the coronavirus disease 2019 (COVID-19) outbreak in China: summary of a report of 72314 cases from the Chinese Center for Disease Control and Prevention. JAMA. https://doi.org/10. 1001/jama.2020.2648

22. Zhou F, Yu T, Du R et al (2020) Clinical course and risk factors for mortality of adult inpatients with COVID-19 in Wuhan, China: a retrospective cohort study. Lancet 395:1054-1062

23. Guo W, Li M, Dong Y et al (2020) Diabetes is a risk factor for the progression and prognosis of COVID-19. Diabetes Metab Res Rev. https://doi.org/10.1002/dmrr.3319:e3319

24. Tan L, Wang Q, Zhang D et al (2020) Lymphopenia predicts disease severity of COVID-19: a descriptive and predictive study. Signal Transduct Target Ther 5:33

25. Bernheim A, Mei X, Huang $M$ et al (2020) Chest CT findings in coronavirus disease-19 (COVID-19): relationship to duration of infection. Radiology. https://doi.org/10.1148/radiol.2020200463: 200463

26. Wu J, Wu X, Zeng $\mathrm{W}$ et al (2020) Chest CT findings in patients with coronavirus disease 2019 and its relationship with clinical features. Invest Radiol 55:257-261

27. Zhou Z, Guo D, Li C et al (2020) Coronavirus disease 2019: initial chest CT findings. Eur Radiol. https://doi.org/10.1007/s00330-02006816-7

28. Ye Z, Zhang Y, Wang Y, Huang Z, Song B (2020) Chest CT manifestations of new coronavirus disease 2019 (COVID-19): a pictorial review. Eur Radiol. https://doi.org/10.1007/s00330-02006801-0
29. Minodier L, Masse S, Capai L et al (2017) Clinical and virological factors associated with gastrointestinal symptoms in patients with acute respiratory infection: a two-year prospective study in general practice medicine. BMC Infect Dis 17:729

30. Wan Y, Shang J, Graham R, Baric RS, Li F (2020) Receptor recognition by the novel coronavirus from Wuhan: an analysis based on decade-long structural studies of SARS coronavirus. J Virol 94

31. D'Amico F, Baumgart DC, Danese S, Peyrin-Biroulet L (2020) Diarrhea during COVID-19 infection: pathogenesis, epidemiology, prevention and management. Clin Gastroenterol Hepatol. https:// doi.org/10.1016/j.cgh.2020.04.001

32. $\mathrm{Xu} \mathrm{H}$, Zhong L, Deng J et al (2020) High expression of ACE2 receptor of 2019-nCoV on the epithelial cells of oral mucosa. Int J Oral Sci 12:8

33. Zou X, Chen K, Zou J, Han P, Hao J, Han Z (2020) Single-cell RNA-seq data analysis on the receptor ACE2 expression reveals the potential risk of different human organs vulnerable to 2019-nCoV infection. Front Med. https://doi.org/10.1007/s11684-020-0754-0

34. Gu J, Han B, Wang J (2020) COVID-19: gastrointestinal manifestations and potential fecal-oral transmission. Gastroenterology. https://doi.org/10.1053/j.gastro.2020.02.054

35. Roman GC, Spencer PS, Reis J et al (2020) The neurology of COVID-19 revisited: a proposal from the Environmental Neurology Specialty Group of the World Federation of Neurology to implement international neurological registries. J Neurol Sci 414:116884

36. Peiris JS, Chu CM, Cheng VC et al (2003) Clinical progression and viral load in a community outbreak of coronavirus-associated SARS pneumonia: a prospective study. Lancet 361:1767-1772

37. Booth CM, Matukas LM, Tomlinson GA et al (2003) Clinical features and short-term outcomes of 144 patients with SARS in the greater Toronto area. JAMA 289:2801-2809

38. Who Mers-Cov Research Group (2013) State of knowledge and data gaps of Middle East respiratory syndrome coronavirus (MERS-CoV) in humans. PLoS Curr 5

39. Chai X, Hu L, Zhang Y et al (2020) Specific ACE2 expression in cholangiocytes may cause liver damage after 2019-nCoV infection. bioRxiv. https://doi.org/10.1101/2020.02.03.931766

Publisher's note Springer Nature remains neutral with regard to jurisdictional claims in published maps and institutional affiliations. 